\section{POS1441 SYMPTOMS CHARACTERISTICS OF SEROPOSITIVE INDIVIDUALS AT-RISK FOR DEVELOPING RHEUMATOID ARTHRITIS ARE VERSATILE AND COMPARABLE TO THOSE IN PEOPLE WITH EARLY RHEUMATOID ARTHRITIS}

P. Studenic ${ }^{1,2}$, A. Circiumaru ${ }^{1,3}$, D. Aletaha ${ }^{2}$, K. Chatzidionysiou ${ }^{1,4}$, A. Hensvold ${ }^{1,3}$, A. Catrina ${ }^{1,4} .{ }^{1}$ Karolinska Institute, Department of Medicine (Solna), Division of Rheumatology, Stockholm, Sweden; ${ }^{2}$ Medical University of Vienna, Department of Internal Medicine 3, Division of Rheumatology, Wien, Austria; ${ }^{3}$ Academic Specialist Center - Stockholm Health Care Services, Center for Rheumatology, Stockholm, Sweden; ${ }^{4}$ Karolinska University Hospital, Rheumatology Unit, Stockholm, Sweden

Background: The symptom burden of typical rheumatoid arthritis (RA) symptoms in those at risk for developing RA - positive for anti-citrullinated peptide antibodies (ACPA) and musculoskeletal complaints - has not been explored. Objectives: To evaluate patient-reported symptoms in individuals at-risk to develop RA in comparison to people with newly diagnosed, early seropositive RA.

Methods: Two datasources from the region of Stockholm were used: The RISK RA cohort follows up ACPA positive individuals with arthralgia, but without signs of joint inflammation in a structured program over 3 years. The baseline visit of this cohort was used. Patients with early seropositive (ACPA and/or rheumatoid factor positive) RA (symptom duration of 12 months maximum) reported in the Swedish Rheumatology Quality Register (SRQ) at their closest visit to their diagnosis date before receiving DMARD treatment have been identified. At-risk individuals were matched $1: 3$ by sex and age using the nearest neighbour method utilizing Mahalanobis distance, corrected for sample bias and exact matches on sex. Effect estimates of being at-risk compared to early RA for pain, patient global $(\mathrm{GH})$, fatigue (all visual analogue scales), health assessment questionnaire (HAQ), TJC28 and the EuroQol-5D (EQ5D; range: 0-1) have been derived. Propensity score matching was used as sensitivity analyses.

Results: A total of 223 individuals at risk for developing RA were compared to 820 matched early RA patients. The summary of distribution of variables and effect estimates of differences between at-risk and early RA individuals are shown in the Figure 1. At-risk individuals show $24 \mathrm{~mm}$ of lower pain scores than early RA. This difference is even less $(-17 \mathrm{~mm}, 95 \% \mathrm{Cl}$ : -24 to -11$)$ when the estimate is additionally matched for the number of tender joints. The TJC28 was on average 5.4 joints lower than in early RA patients. People at-risk show $22 \mathrm{~mm}$ lower GH scores (rate themselves better) and $17 \mathrm{~mm}$ lower fatigue scores than early RA patients. Fatigue was scored highest (mean:35mm, 95\%Cl: 30 to 29) among the three VAS. HAQ was on average lower by 0.6 points in at-risk individuals and EQ5D showed 0.24 higher index-values, outlining a better health status. Still the mean EQ5D in at-risk individuals was only $0.74(95 \% \mathrm{Cl}: 0.71$ to 0.77$)$, which is lower than average values of an age-matched general population (0.92 to 0.96$)$. Sensitivity analyses revealed similar results.

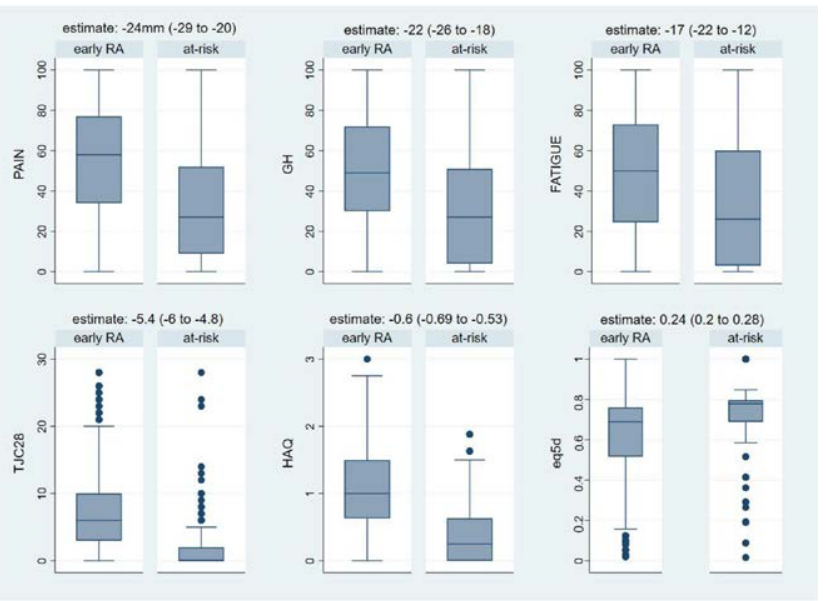

Figure 1. Boxplots of pain, global health, fatigue, TJC28, EQ5D and HAQ, separately displayed for people with early seropositive RA and individuals at-risk. Population effect estimates (all $p<0.001$ ) comparing at-risk to early RA are provided for every pair, with $95 \%$ confidence interval in brackets.

Conclusion: Not surprisingly, individuals at risk for RA report less symptom burden then early diagnosed RA patients. However, these differences only range around minimal clinically important differences and for fatigue even below, which stresses the need for medical attention and management strategies for symptomatic at-risk individuals.
Acknowledgements: This study was supported through the New Horizon Fellowship and the FOREUM research fellowship grant and is part of the Innovative Medicines Initiative Joint Undertaking under grant agreement no 777357 (RTCure).

Hensvold $\mathrm{AH}$ and Catrina IA contributed equally.

Disclosure of Interests: None declared

DOI: 10.1136/annrheumdis-2021-eular.3884

\section{Validation of outcome measures and biomarkers}

\begin{tabular}{|l|l|}
\hline POS1442 & THE SUBGROUP OF ACPA-NEGATIVE RHEUMATOID \\
ARTHRITIS PATIENTS ACHIEVING SUSTAINED \\
DMARD-FREE REMISSION IS CHARACTERIZED BY A \\
DIFFERENT RESPONSE IN SEROLOGICAL MARKERS \\
WITHIN THE FIRST YEAR OF DMARD-INITIATION
\end{tabular}

M. Verstappen ${ }^{1}$, A. Van der Helm - van Mil ${ }^{1,2} .{ }^{1}$ Leiden University Medical Center, Rheumatology, Leiden, Netherlands; ${ }^{2}$ Erasmus University Medical Center, Rheumatology, Rotterdam, Netherlands

Background: Whilst $\sim 40 \%$ of ACPA-negative RA-patients achieve sustained DMARD-free remission (SDFR; the sustained absence of synovitis after DMARD-cessation), the biological pathways involved in SDFR-development are incompletely understood. Previously, high baseline levels of MMP-3, SAA and CRP were found in ACPA-negative RA-patients achieving SDFR. Differences in biomarker levels might reflect distinct underlying biological pathways leading towards disease resolution in this subgroup of ACPA-negative RA-patients. Subsequently, the course of specific biomarkers after treatment-initiation could attain more insight in the understanding of SDFR-development in ACPA-negative RA. Objectives: We explored whether the response of specific biomarkers after treatment-initiation could increase the understanding of SDFR-development in ACPA-negative RA

Methods: One-hundred-thirty-one consecutive ACPA-negative RA-patients were studied on SDFR-development during (median) 7.2-years follow-up. Twelve biomarkers (SAA/CRP/MMP-1/MMP-3/resistin/leptin/IL-6/TNF-R1/YKL 40/EGF/VEGF/VCAM-1) were measured at diagnosis and 1-, and 2-years after DMARD-initiation and compared between patients achieving and not achieving SDFR. To extend on previous findings on baseline levels, correlations between baseline levels and change in levels within year-1 were determined using spearman's rho $(\rho)$.

Results: RA-patients that achieved SDFR $(n=63)$ were characterized by a stronger decline in MMP-1, MMP-3, SAA and CRP in the first 12-months after DMARD-start, compared to RA-patients not achieving SDFR. MMP-1 and MMP-3 declined 1.30-times $(95 \% \mathrm{Cl} ; 1.08-1.57)$ and 1.44 -times $(95 \% \mathrm{Cl} ; 1.00$ 2.06) stronger in SDFR-patients. In SAA and CRP, this decline was 2.12-times $(95 \% \mathrm{Cl} ; 1.08-4.14)$ and $2.24-$ times $(95 \% \mathrm{Cl} ; 1.16-4.35)$ stronger. Baseline levels of MMP-3, SAA and CRP and strongly correlated with decline in 12-months; $\rho$ $-0.6991, \rho-0.9205$ respectively $\rho-0.9008$. RA-patients with high baseline levels characterized the subgroup with the strongest decline in biomarker levels in the first year after DMARD-initiation.

Conclusion: The subgroup ACPA-negative RA-patients achieving SDFR are characterized by higher baseline levels and a stronger serological response of MMP-3, SAA and CRP after DMARD-initiation. Because MMP-3 and SAA are expressed by fibroblast-like synoviocytes, these cells may differ in RA-patients achieving disease resolution; molecular studies on synovial tissue could be a next step.

Disclosure of Interests: None declared

DOI: 10.1136/annrheumdis-2021-eular.246

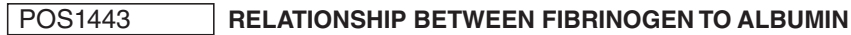 RATIO AND DISEASE ACTIVITY IN PATIENTS WITH SYSTEMIC LUPUS ERYTHEMATOSUS}

M. Liu ${ }^{1}$, Y. Huang ${ }^{1}$, Z. Huang ${ }^{1}$, Q. Huang ${ }^{1}$, T. W. Li ${ }^{1}$. ${ }^{1}$ Guangdong Second Provincial General Hospital, Department of Rheumatology and Immunology, Guangzhou, China

Background: Systemic lupus erythematosus (SLE) is a systemic inflammatory autoimmune disease characterized by excessive production of immune complexes and proinflammatory cytokine. Low complement, neutrophil to lymphocyte ratio (NLR) and platelet to lymphocyte ratio (PLR) have been used as inflammatory biomarkers to assess the disease activity of SLE. Recently, the fibrinogen to albumin ratio (FAR) has emerged as an effective indicator to reflect the systemic inflammation in many diseases. However, the role of FAR in SLE has been scarcely studied.

Objectives: This study was to investigate the association between FAR and SLE Disease Activity Index 2000 (SLEDAI-2K) in SLE. 